\title{
5 Research Square

\section{Application of Ex Vivo Liver Resection Followed by the Autotransplantation Technique in Radical Resection of Gastric Cancer Liver Metastases: A Case Report}

\section{Hong Wang ( 976411979@qq.com)}

Third Military Medical University Southwest Hospital https://orcid.org/0000-0003-1396-4098

Chengcheng Zhang

Third Military Medical University Southwest Hospital

\section{Yanjiao Ou}

Third Military Medical University Southwest Hospital

Leida Zhang (D2518569931@qq.com)

Third Military Medical University Southwest Hospital

\section{Case report}

Keywords: Ex-vivo Liver Resection Followed by Autotransplantation Technique, Gastric Cancer Liver Metastases

Posted Date: September 3rd, 2020

DOI: https://doi.org/10.21203/rs.3.rs-38771/v2

License: (c) (1) This work is licensed under a Creative Commons Attribution 4.0 International License. Read Full License 


\section{Abstract}

Background Radical resection of gastric cancer liver metastases can increase the 5-year survival rate of GCLM patients. However, patients may lose the theoretical feasibility of surgery due to the critical location of liver metastasis in some cases.

Case presentation A 29-year-old female had a chief complaint of chronic abdominal pain for one year. Abdominal CT and MRI examinations suggested a mass of unknown pathological nature located between the first and second hila and the margin of the lower segment of the right lobe of the liver. The anterior wall of the gastric antrum was unevenly thickened. The diagnosis of (gastric antrum) intramucosal well-differentiated adenocarcinoma was histopathologically confirmed by puncture biopsy with gastroscopy guidance. The pathological properties of the liver mass remained unclear before

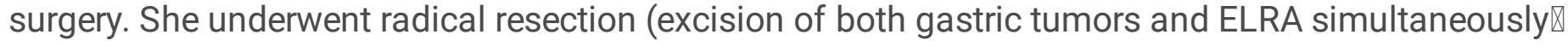
followed by XELOX adjuvant chemotherapy. Without serious postoperative complications, the patient was successfully discharged on the 20th day after the operation. Pathological examination of the excised specimen indicated that gastrectomy with $\mathrm{D} 2$ lymph node dissection for primary gastric tumors and R0 resection for liver metastases were achieved. The resected mass was confirmed to be poorly differentiated gastric carcinoma (hepatoid adenocarcinoma with neuroendocrine differentiation) with liver metastases in segments 6 and 8. A Follow-up review that began one month after surgery showed no recurrence or metastasis within the liver. The mother of three children miraculously lived a happy life and went back to work as normal.

Conclusions: Application of ELRA in radical resection for GCLM can help selected patients with intrahepatic metastases located in complex sites obtain a favorable clinical outcome.

\section{Introduction}

Previous studies have reported that the incidence of GCLM ranges from $9.9 \%$ to $18.7 \%(1,2)$, with a median survival time of 11 months and a 5 -year survival rate $<20 \%(3)$. Although retrospective studies in multiple centers have declared that radical resectionखexcision of both gastric tumors and liver metastases

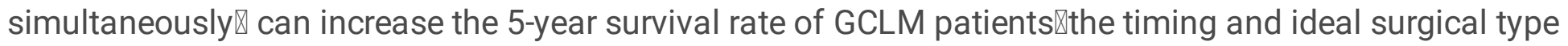
still remain controversial. Based on the likelihood of a surgical treatment being successfulखC-GCLM has been proposed by the Chinese consensus on the diagnosis and treatment of GCLM(4). While we acknowledge a tumor-free margin as the predictor of a better outcome, a unavoidable question may emerge $\varangle$ what if liver metastasis meets all the surgical qualifications judged by size and scope according to the aforementioned classification criteria, but their location is so critical that the technical risk of surgery significantly increases? It would be regretful to give up the chance of radical resection because of the limited number of eligible patients. The application of ELRA may provide an alternative solution to this problem. Here, we present a case pertaining to the treatment procedures and prognosis of a young female patient with GCLM $₫$ that showed that the application of ELRA in radical resection for GCLM can help patients with intrahepatic metastases located in complex sites obtain a favorable clinical outcome. 


\section{Case Presentation}

\subsection{Preoperative assessment}

The patient was a 29-year-old female had a chief complaint of chronic abdominal pain for one year and she experienced continuous weight loss of $5 \mathrm{~kg}$ over 2 months prior to her admission. According to her past medical history, she underwent ovarian cystectomy, appendectomy and subtotal hysterectomy for uncontrollable hemorrhage. No special family history was provided except that her mother was diagnosed with squamous cell carcinoma of the vulvar epithelium and the disease was cured according to her description. Other detailed basic information is displayed in Table 1. Upon physical examination, a round mass approximately $7 \mathrm{~cm}$ in diameter could be palpated in the right upper abdomen and the mass was smooth, pushable, hard and indistinguishable from adjacent tissue. Abdominal CT and MRI examination suggested a mass of unknown pathological nature located between the first and second hila

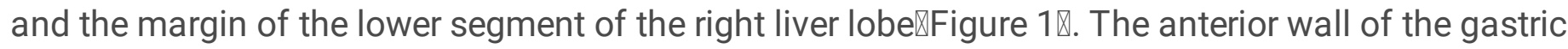
antrum was unevenly thickened. PET/CT (whole body) examination showed two main points: 1. the anterior wall of the gastric antrum was unevenly thickened, and FDG uptake was increased, which was consistent with the manifestations of gastric cancer; and 2. a mass shadow was located at the first and second hepatic hila and the lower right lobe of the liver. As increased FDG uptake is a sign of malignancy, whether the mass was liver metastasis or primary liver cancer remained to be identified. The results of puncture biopsy with gastroscopy guidance supported the pathological diagnosis of gastric antrum intramucosal well-differentiated adenocarcinoma. ECG, chest DR and CT of the lower abdomen and pelvis showed no obvious abnormalities. Laboratory data demonstrated normal liver function and routine blood test results囚Table2】. The tumor marker results showed that AFP was $9850 \mathrm{ng} / \mathrm{ml}$ and CEA was 55 $\mathrm{ng} / \mathrm{ml}$. Preoperative volumetric calculation was achieved by using 3D visualization imaging. The remnant liver-to-standard liver volume ratio was $56 \%$. ICGR15 was $2.1 \%$. The preoperative Child-Pugh score was 3. Due to the unknown nature and special location of the mass in the liver, it was considered to be a liver metastatic focus of gastric cancer after consultation by general and hepatobiliary surgeons. After fully understanding the risk and difficulty of the operation and after careful consideration, the patient decided to accept ELRA combined with simultaneous radical resection for gastric cancer.

\subsection{Surgical procedure}

A tumor protruding through the liver capsule with unclear borders and an uneven surface was found in the right liver during abdominal exploration. It was hard and pale and was tightly adhered to the surrounding omentum. Consistent with the preoperative imaging findings, the tumor extended to the junction of the left and right liver lobes and was adjacent to the vena cava. Intraoperative frozen biopsy of the liver mass was performed and the results supported a poorly differentiated malignant tumor. The presence of intrahepatic microlesions was ruled out by intraoperative ultrasound. The results of intraoperative exploration and the desire of the patient led us to perform ELRA combined with radical resection for gastric cancer as the original plan. 
Hepatectomy was accomplished by using standard technique (5) with extensive lymph node dissection around the SMA and celiac trunk. The common bile duct was completely removed up to the head of the pancreas. Preservation and perfusion of the removed isolated liver was performed with ice-cold solution at $4{ }^{\circ} \mathrm{C}$ and UW solution via the intact portal vein. At the same time, temporary channel was established between the SHIVC and IHIVC with an internal diameter of $2 \mathrm{~cm}$ and Geo-Tex artificial vessel. The PV was also anastomosed to the vessel to construct portacaval shunt. After the intrahepatic tumor and target liver segment were completely resected, the aforementioned temporary venous channel was removed, and the remnant of the liver was implanted similar to OLT which was followed by anastomosis of the SHIVC, IHIVC, PV, HA, and bile duct. The total anhepatic period was 245 minutes: including 180 minutes of CIT for extracorporeal tumor resection and vein repair, 40 minutes of vena cava anastomosis and 25 minutes of portal vein anastomosis. Observation of the normal liver surface color after reperfusion was commonly performed, and the surgeons usually confirmed bile outflow before bile duct anastomosis. Some of intraoperative photos are presented in Figure 1. As we performed ex vivo liver resection on the back table, radical surgery for gastric cancer was accomplished simultaneously by general surgeons on the operating table.

\subsection{Postoperative management and follow-up}

Our medical team provided her with symptomatic treatment after surgery such as gastrointestinal decompression, abdominal drainage, anti-infection medication, nutritional support. She underwent bile leakage drainage on the fifth day after the operation. Fluid accumulated in the liver section and caused symptoms of gastric compression. The effusion was completely drained out after CT-guided puncture and catheter drainage. No other postoperative complications such as SFSS, ALF, vascular embolism, intra-abdominal infection, or hemorrhage occurred. She was successfully discharged on the 20th day after the operation. Postoperative pathological examination of the excised specimen indicated that gastrectomy with $\mathrm{D} 2$ lymph node dissection for primary gastric tumors and R0 resection for liver metastases were achieved. The resected mass was confirmed to be poorly differentiated gastric carcinoma (hepatoid adenocarcinoma with neuroendocrine differentiation) with liver metastases in segments of 6 and 8 . The patient began her first follow-up one month after the operation, and received 6 courses of XELOX chemotherapy, during which no serious adverse reactions or treatment discontinuation occurred. No obvious signs of tumor recurrence or metastasis within the abdomen or liver were recorded during a 3.5-year follow-up. The patient had a good overall condition and did not undergo a second operation. Selected pre and post operative abdominal CT images of the patient were displayed in Figure 2. Postoperative laboratory data of the patient were displayed in Table 3.

\section{Discussion And Conclusions}

As an advanced-stage type of gastric cancer冈GCLM is usually translobal, multifocal or even diffusely spread, and complicated by peritoneal, extensive lymph node and organ metastasis due to its highly malignant and rapidly invasive oncologic nature. Poor prognosis and deadly surgical complications make the resection rate so low that there is no multicenter clinical trial with large samples on the 
significance of liver resection for GCLM. No well-established criteria exist except for a few retrospective studies with a limited number of cases(4). However, it is worth noting that the results of these limited studies almost indicate that patients with GCLM can benefit from radical resection (6-9). According to follow-up results, the patient we reported also obtained a good prognosis and lived with a good quality of life.

According to the C-GCLM, the definitions of both type I and II include the same criterion:"technological resectability of liver metastases judged by a hepatobiliary surgeon". It emphasizes the principle that surgery should be performed only when R0 resection is anticipated(4). The aforementioned question arises: what if the size and scope of liver metastases comply with this standard, but the anatomical complexity puts the hepatobiliary surgeon into a dilemma? The most common situation is that the tumor is located deeply within the liver and has extensive involvement with the main hepatic veins or retrohepatic vena cava. Conventional radical resection is extremely hazardous due to the potential risk of uncontrollable hemorrhage and long ischemia time(10). These types of tumors are usually deemed unresectable because they can not be completely removed with conventional surgery(11) .

We can infer that ELRA may overcome this issue from our case report. It effectively alleviated the technical bottleneck caused by the special location of the tumor in traditional hepatectomy and allowed the hepatobiliary surgeons to perform precise liver tumor resection and effective vascular/repair by enabling them to operate with bloodless vision and access to critical structures easily since the first report of ex vivo hepatectomy by Pichlmayr et al in 1988(4, 12, 13).

The theoretical basis and detailed surgical procedures that have been reported systematically in many studies will not be repeated here(14). What we want to discuss is the subtle principle of contradiction》 with greater benefit comes higher risk. From the surgical records described above, we can see that ELRA is a challenging and time-consuming surgery with cumbersome procedures and complex operations. As a

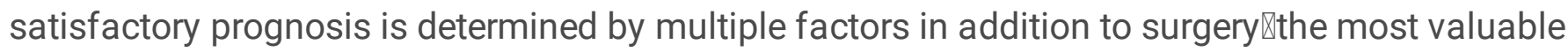
experience our team has summarized from this case is that accurate preoperative evaluation for specific patients based on detailed medical examination is of great importance. Strict patient selection and precise assessment of the size and quality of the remnant liver are pivotal to the decision-making process(14). Although experiences gained from previous studies have indicated that after extended hepatectomy involving 70 to $75 \%$ of the liver, the remnant liver can still function well in non-cirrhosis patients $(15,16)$, the strategy may need to be adjusted in the application of ERLA according to the specific situation. A major feature of ERAT is the longer CIT compared with ordinary LT(17). In addition, ERAT procedures are more complex than LT and hepatectomy so a longer operative time and more intraoperative blood loss seem inevitable(10). These are two poor prognostic indicators for LT recipients and hepatectomy patients because they may lead to graft loss due to the high incidence of postoperative biliary and arterial complications or severe hepatic dysfunction(18). Based on the above theory, the liver function, quality and estimated RLV of ELRA patients need to meet higher requirements. Livers with poor quality are less tolerant to cold ischemia-reperfusion injury. In addition, sufficient preoperative preparations, such as biliary drainage in patients with obstructive jaundice and nutritional support in 
malnourished patients, are indispensable once the doctor and patient reach a consensus to perform the surgery. Without exaggeration, it can be considered that half the success will be achieved if suitable GCLM patients are screened out, but inaccurate preoperative assessments will force surgeons to discontinue the operation or will even cause fatal postoperative complications such as SFSS or ALF(19). Aji T and Dong JH et al described the largest case series of 69 patients with end-stage hepatic AE who underwent ELRA.The detailed methods and procedures applied to select patients preoperatively are explicitly described in the literature. We also adopted the same issues to evaluate the patient in this case report(14).

With greater benefit comes higher risk. Hepatobiliary surgeons must pursue more accurate and rapid surgical techniques to shorten the CIT and anhepatic phase. Under the condition of the liver in vitro, full exposure of the surgical area can help reduce the risk due to vascular or bile duct variation, which may not be detected before the operation. A longer anhepatic period can lead to circulatory and metabolic disorders. This is also a major test for the monitoring and intraoperative management of the anesthesiology department. Launching this type of major surgery can help improve the overall medical level of institutions and optimize the multidisciplinary diagnosis and treatment model. The lack of consensus and guidance on the application of ELRA drives us to accumulate more successful cases and precious experiences. For instance, Yiwen Qiu et al proposed their vascular infiltration-based classification as a tool to improve anatomic comprehension and facilitate surgical planning for ELRA(20). With the continuous accumulation of experience and the gradual improvement of technology, many so-called surgical penalty areas will also be broken through. The favorable implementation of this surgery can also enable surgeons to build confidence in dealing with more complex situations. Once successful, it will bring great significance in two aspects. For patients and their families, the painful clinical symptoms caused by the tumor will be controlled, and the quality of life will be dramatically improved. They will see improvements in mood and will achieve a better physical state that remains for the rest of their lives. From a medical point of view, an increased survival rate may provide a longer time for more postoperative treatment options and promote the progress of clinical research.

We must admit the limitation that many diseases, such as advanced cancer, still cannot be completely cured, although medical science has made rapid progress in recent years. However, it is a responsibility and obligation to create an opportunity for patients who never give up on themselves to extend their lives to fulfill their unfinished wishes. To a certain extent, this is also the meaning and motivation to encourage us to explore and push the surgical boundary. During the disease-free survival period, the female patient in our case spent irreplaceable days with her three cute children who filled her life with happiness and hope. She was deeply grateful for her brave choice and expressed her endless gratitude to our team.

In conclusion, the application of ELRA in radical resection of GCLM, as our case shows, could provide an alternative for selected patients. Surgeons'technical expertise combined with careful patient selection and perioperative management guaranteed by an experienced MDT could contribute to favorable clinical outcomes. 


\section{Declarations}

\section{Ethics approval and consent to participate}

This report was carried out in accordance with the principles of the Declaration of Helsinki.

\section{Consent for publication}

Written informed consent was obtained from the patient for publication of this case report and accompanying images and clinical data.

\section{Availability of data and materials}

The datasets used in the report are available from the corresponding author on reasonable request.

\section{Competing interests}

The authors declare that they have no competing interests.

\section{Funding}

The authors declare that this work was not supported by any grants or funding.

\section{Authors'contributions}

Hong Wang wrote the manuscript. Chengcheng Zhang helped writing the manuscript. All authors administered the daily medical treatments in this case. Leida Zhang comprehensively supervised this study. All authors read and approved the final manuscript.

\section{Acknowledgements}

All images were taken and post-processed by a professional photographer in our center

\section{Abbreviations}

GCLM: gastric cancer liver metastases $₫$ C-GCLM $₫$ Chinese type for gastric cancer liver metastases $₫ E L R A$

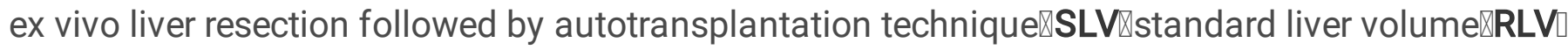

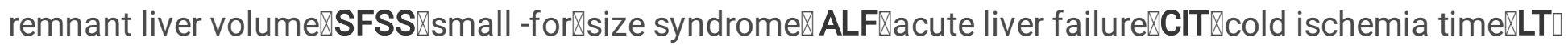

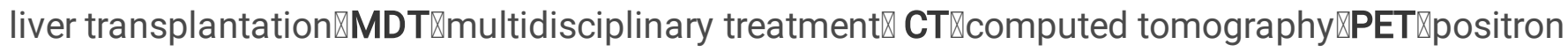

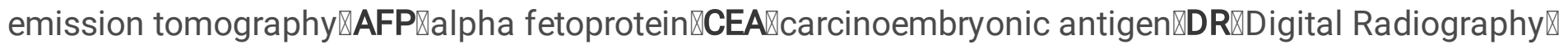
FDG $₫ \beta$-2-[ 18 F]-Fluoro-2-deoxy-D-glucose $\triangle P E T / C T$ positron emission tomography/ computed

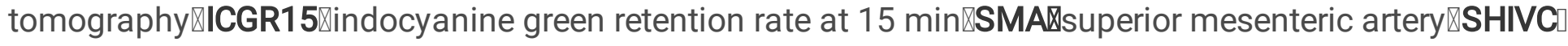

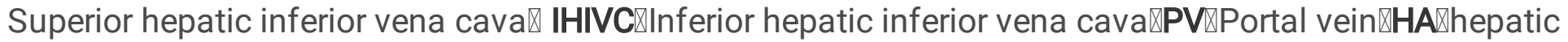
artery 


\section{References}

1. Riihimäki M, Hemminki A, Sundquist $K$, Sundquist J, Hemminki K. Metastatic spread in patients with gastric cancer. Oncotarget. 2016;7(32):52307-16. doi:10.18632/oncotarget.10740

2. Cheon SH, Rha SY, Jeung HC, Im CK, Kim SH, Kim HR, et al. Survival benefit of combined curative resection of the stomach (D2 resection) and liver in gastric cancer patients with liver metastases. Annals of oncology : official journal of the European Society for Medical Oncology. 2008;19(6):114653. doi:10.1093/annonc/mdn026

3. Xiao Y, Zhang B, Wu Y. Prognostic analysis and liver metastases relevant factors after gastric and hepatic surgical treatment in gastric cancer patients with metachronous liver metastases: a population-based study. Irish journal of medical science. 2019;188(2):415-24. doi:10.1007/s11845018-1864-4

4. Zhang K, Chen L. Chinese consensus on the diagnosis and treatment of gastric cancer with liver metastases. Therapeutic advances in medical oncology. 2020;12:1758835920904803. doi:10.1177/1758835920904803

5. Kaishan T, Dong C, Zhaoxu Y, Xiao L, Ying T. Chinese Code of practice for liver Transplantation (2019 Edition)( J ) Chinese Journal of Transplantation(Electronic Version). 2019;13(03):171-6. doi:10.3877/cma.j.issn.1674-3903.2019.03.003

6. Kunieda K, Saji S, Sugiyama Y, Osada S, Sano J, Nagao N, et al. Evaluation of treatment for synchronous hepatic metastases from gastric cancer with special reference to long-term survivors. Surgery today. 2002;32(7):587-93. doi:10.1007/s005950200106

7. Ueda K, Iwahashi M, Nakamori M, Nakamura M, Naka T, Ishida K, et al. Analysis of the prognostic factors and evaluation of surgical treatment for synchronous liver metastases from gastric cancer. Langenbeck's archives of surgery. 2009;394(4):647-53. doi:10.1007/s00423-008-0311-9

8. Thelen A, Jonas S, Benckert C, Lopez-Hänninen E, Neumann U, Rudolph B, et al. Liver resection for metastatic gastric cancer. European journal of surgical oncology : the journal of the European Society of Surgical Oncology and the British Association of Surgical Oncology. 2008;34(12):1328-34. doi:10.1016/j.ejso.2008.01.022

9. Petrelli F, Coinu A, Cabiddu M, Ghilardi M, Borgonovo K, Lonati V, et al. Hepatic resection for gastric cancer liver metastases: A systematic review and meta-analysis. Journal of surgical oncology. 2015;111(8):1021-7. doi:10.1002/jso.23920

10. Shen S, Qiu Y, Yang X, Wang W. Remnant Liver-to-Standard Liver Volume Ratio Below $40 \%$ is Safe in Ex Vivo Liver Resection and Autotransplantation. Journal of gastrointestinal surgery : official journal of the Society for Surgery of the Alimentary Tract. 2019;23(10):1964-72. doi:10.1007/s11605-0184022-4

11. Moris D, Tsilimigras DI, Chakedis J, Beal EW, Felekouras E, Vernadakis S, et al. Liver transplantation for unresectable colorectal liver metastases: A systematic review. Journal of surgical oncology. 2017;116(3):288-97. doi:10.1002/jso.24671 
12. Zhanyu Y, Qian L, Xiangde L, Zhiqing Y, Tengqian T, Ping B. Ex-vivo liver resection combined partial liver autotransplantation for hepatocellular carcinoma located at critical site $(\mathrm{J})$ Chinese Journal of Digestive Surgery. 2010(01):18-20. doi:10.3760/cma.j.issn.1673-9752.2010.01.009

13. Pichlmayr R, Bretschneider HJ, Kirchner E, Ringe B, Lamesch P, Gubernatis G, et al. [Ex situ operation on the liver. A new possibility in liver surgery]. Langenbecks Archiv fur Chirurgie. 1988;373(2):122-6. doi:10.1007/BF01262775

14. Aji T, Dong JH, Shao YM, Zhao JM, Li T, Tuxun T, et al. Ex vivo liver resection and autotransplantation as alternative to allotransplantation for end-stage hepatic alveolar echinococcosis. Journal of hepatology. 2018;69(5):1037-46. doi:10.1016/j.jhep.2018.07.006

15. Pak LM, Chakraborty J, Gonen M, Chapman WC, Do RKG, Groot Koerkamp B, et al. Quantitative Imaging Features and Postoperative Hepatic Insufficiency: A Multi-Institutional Expanded Cohort. Journal of the American College of Surgeons. 2018;226(5):835-43.

doi:10.1016/j.jamcollsurg.2018.02.001

16. Markar SR, Mikhail S, Malietzis G, Athanasiou T, Mariette C, Sasako M, et al. Influence of Surgical Resection of Hepatic Metastases From Gastric Adenocarcinoma on Long-term Survival: Systematic Review and Pooled Analysis. Annals of surgery. 2016;263(6):1092-101.

doi:10.1097/SLA.0000000000001542

17. Rammohan A, Govil S, Vargese J, Kota V, Reddy MS, Rela M. Changing pattern of biliary complications in an evolving liver transplant unit. Liver transplantation : official publication of the American Association for the Study of Liver Diseases and the International Liver Transplantation Society. 2017;23(4):478-86. doi:10.1002/It.24736

18. Ikegami T, Yoshizumi T, Sakata K, Uchiyama H, Harimoto N, Harada N, et al. Left lobe living donor liver transplantation in adults: What is the safety limit? Liver transplantation : official publication of the American Association for the Study of Liver Diseases and the International Liver Transplantation Society. 2016;22(12):1666-75. doi:10.1002/It.24611

19. Haiwen Y, Ying Z, Qian L, Lingqiang Z, Yong D, Xiaolei X, et al. Small-for-size syndrome in hepatic alveolar echinococcosis treated by ex vivo liver resection and autotransplanta-tion:A case report $(\mathrm{J})$ Journal of Clinical Hepatology. 2019;35(04):858-60. doi:10.3969/j.issn.1001-5256.2019.04.030

20. Qiu Y, Yang X, Shen S, Huang B, Wang W. Vascular infiltration-based surgical planning in treating end-stage hepatic alveolar echinococcosis with ex vivo liver resection and autotransplantation. Surgery. 2019;165(5):889-96. doi:10.1016/j.surg.2018.11.007

\section{Tables}

\section{Table 1 Detailed Basic Information of the Patient}




\begin{tabular}{|ll|}
\hline item & value \\
Height & $158 \mathrm{CM}$ \\
Weight & $48 \mathrm{KG}$ \\
Obstructive Jaundice & 19.2 \\
Zubrod-ECOG-WHO & Non \\
Hypertension & 1 \\
Diabetes & Non \\
Heart Disease & Non \\
Kidney Disease & Non \\
Liver Disease & Non \\
Breast Disease & Non \\
Genitourinary Diseases & Non \\
Infectious Disease & Non \\
Respiratory Function & Good \\
Mental State & Good \\
Neoadjuvant Chemotherapy & Non \\
\hline
\end{tabular}

Table 2 Preoperative Laboratory Data of The Patient 


\begin{tabular}{|ll|}
\hline item & Value \\
WBC & $6.77 \times 10^{\wedge} 9 / \mathrm{L}$ \\
RBC & $3.62 \times 10^{\wedge} 12 / \mathrm{L}$ \\
Hgb & $111 \mathrm{~g} / \mathrm{L}$ \\
PLT & $168 \times 10^{\wedge} 9 / \mathrm{L}$ \\
Neu & $4.88 \times 10^{\wedge} 9 / \mathrm{L}$ \\
Neu\% & $(\mathrm{Neu} \%) 72.1 \% \uparrow$ \\
ALT & $34 \mathrm{IU} / \mathrm{L}$ \\
AST & $32 \mathrm{IU} / \mathrm{L}$ \\
ALP & $37 \mathrm{IU} / \mathrm{L}$ \\
GGT & $12 \mathrm{IU} / \mathrm{L}$ \\
ALB & $38 \mathrm{~g} / \mathrm{L}$ \\
PALB & $0.15 \mathrm{~g} / \mathrm{L}$ \\
TBA & $16.2 \mathrm{umol} / \mathrm{L}$ \\
DBIL & $6.1 \mathrm{umol} / \mathrm{L}$ \\
IDBIL & $10.1 \mathrm{umol} / \mathrm{L}$ \\
PT & $12.50 \mathrm{sec}$ \\
INR & 0.95 \\
AFP & $9850 \mathrm{ng} / \mathrm{ml}$ \\
CEA & $1.46 \mathrm{ng} / \mathrm{ml}$ \\
\hline
\end{tabular}

Table 3 Postoperative Laboratory Data of The Patient 


\begin{tabular}{|ll|}
\hline item & Value \\
\hline WBC & $5.66 \times 10^{\wedge} 9 / \mathrm{L}$ \\
RBC & $4.07 \times 10^{\wedge} 12 / \mathrm{L}$ \\
Hgb & $121 \mathrm{~g} / \mathrm{L}$ \\
PLT & $154 \times 10^{\wedge} 9 / \mathrm{L}$ \\
Neu & $4.00 \times 10^{\wedge} 9 / \mathrm{L}$ \\
Neu\% & $(\mathrm{Neu} \%) 70.6 \% \uparrow$ \\
ALT & $53.3 \mathrm{IU} / \mathrm{L} \uparrow$ \\
AST & $53.5 \mathrm{IU} / \mathrm{L} \uparrow$ \\
ALP & $113 \mathrm{IU} / \mathrm{L}$ \\
GGT & $17 \mathrm{IU} / \mathrm{L}$ \\
ALB & $42 \mathrm{~g} / \mathrm{L}$ \\
PALB & $0.18 \mathrm{~g} / \mathrm{L}$ \\
TBA & $17.3 \mathrm{umol} / \mathrm{L}$ \\
DBIL & $4.32 \mathrm{umol} / \mathrm{L}$ \\
IDBIL & $12.98 \mathrm{umol} / \mathrm{L}$ \\
PT & $11.40 \mathrm{sec}$ \\
PT-INR & 0.85 \\
AFP & $2.8 \mathrm{ng} / \mathrm{ml}$ \\
CEA & $3.56 \mathrm{ng} / \mathrm{ml}$ \\
\hline
\end{tabular}

Figures 

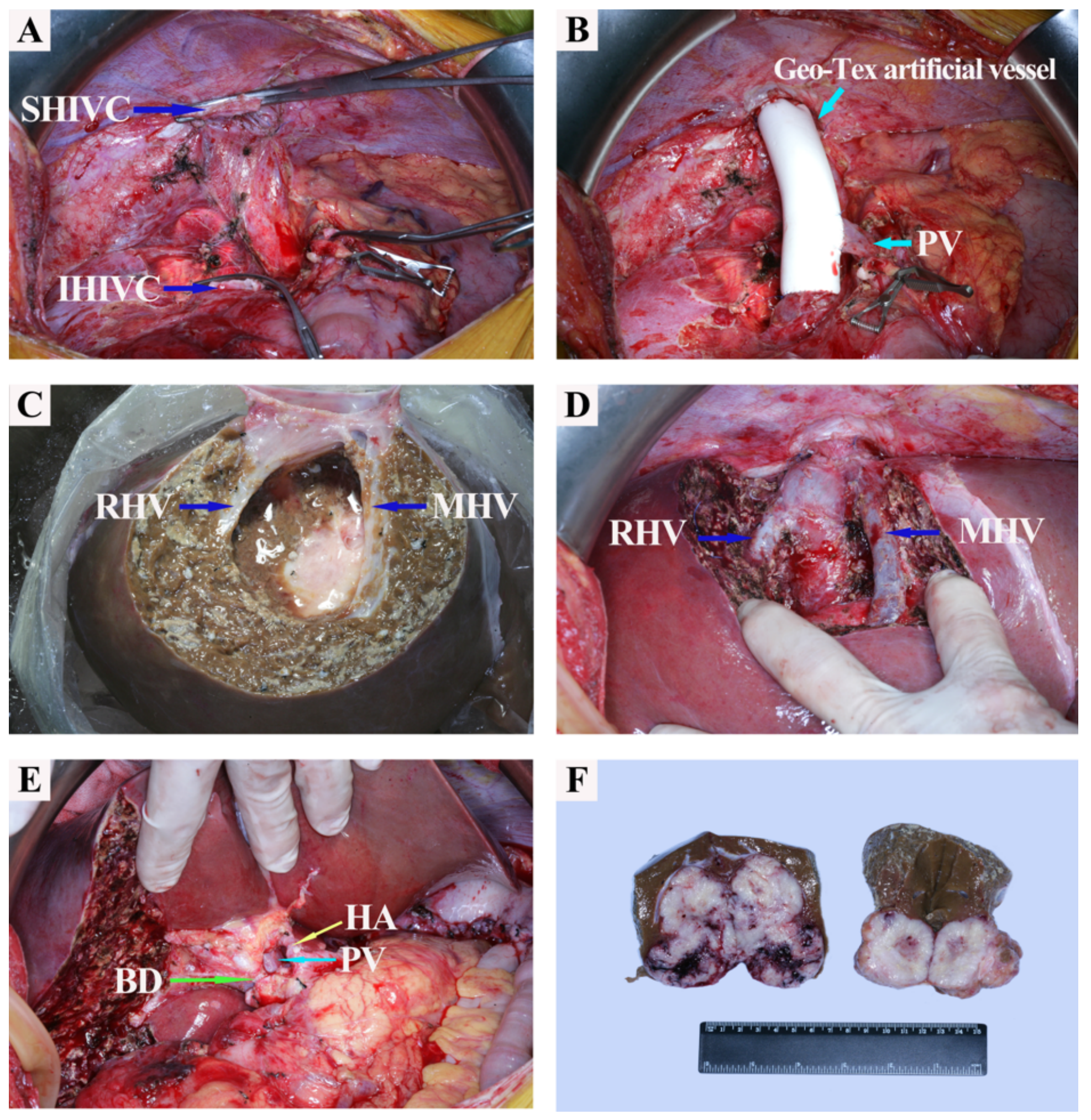

\section{Figure 1}

A. The liver was removed from the abdominal cavity; B. The PV was anastomosed to the Geo-Tex artificial vessel to construct portacaval shunt; $\mathrm{C}$. The tumor has been completely resected from the isolated liver. RHV and MHV was intact; D. The remnant of the liver was implanted similar to OLT. After reconstruction, the RHV, MHV and IVC had unobstructed blood flow; E. The first hepatic hilum after reconstruction; F. The first hepatic hilum after reconstruction. 

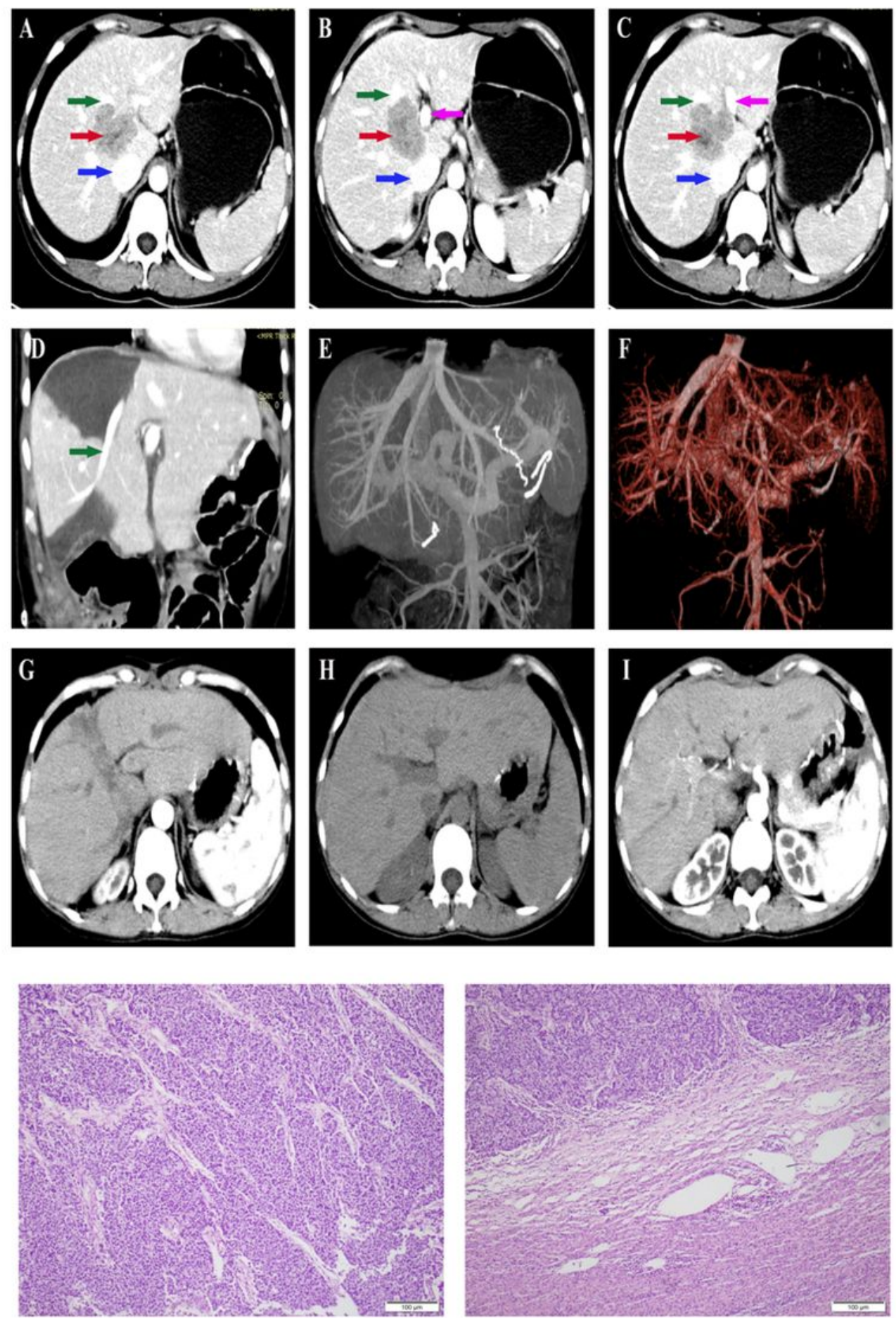

Figure 2

A-C. The tumor冈pointed by the red arrow) was located between the first and second hila and it was

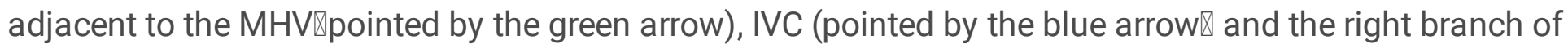

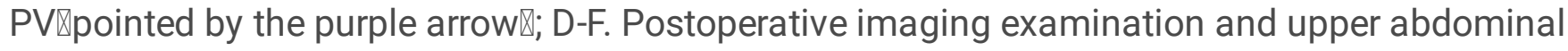
angiography showed that the LHV, MHV, RHV, IVC, HA and PV were intact and Blood flow was unobstructed; G-I. CT results in the first, second and third year after operation showed that there were no 
obvious signs of tumor recurrence. Microscopic section shows poorly differentiated gastric carcinoma (hepatoid adenocarcinoma with neuroendocrine differentiation) with liver metastases

\section{Supplementary Files}

This is a list of supplementary files associated with this preprint. Click to download.

- Incisalmargin.jpg 